\title{
Rad/Nuc Targeted Injury Model
}

National Cancer Institute

\section{Source}

National Cancer Institute. Rad/Nuc Targeted Injury Model. NCI Thesaurus. Code

C161501.

A description of the type of radiation injury or syndrome that is being induced in the animal model. 